\title{
Role of STAT3 and vitamin D receptor in EZH2-mediated invasion of human colorectal cancer
}

\author{
Yan-Wei Lin, I† Lin-Lin Ren, I+ Hua Xiong,' Wan Du,! Ya-Nan Yu,' Tian-Tian Sun,! Yu-Rong Weng,' Zhen-hua \\ Wang,' Ji-Lin Wang,' Ying-Chao Wang,' Yun Cui,' Dan-Feng Sun,' Ze-Guang Han, ${ }^{2}$ Nan Shen, ${ }^{3}$ Weiping Zou, ${ }^{4}$ \\ Jie Xu,' Hao-yan Chen,' Weibiao Cao, ${ }^{5 *}$ Jie Hong ${ }^{1 *}$ and Jing-Yuan Fang ${ }^{1 *}$ \\ I Division of Gastroenterology and Hepatology, Renji Hospital, Shanghai Institution of Digestive Disease; Key Laboratory of Gastroenterology and \\ Hepatology, Ministry of Health; State Key Laboratory of Oncogene and Related Genes, Shanghai liao-Tong University School of Medicine, 145 \\ Middle Shandong Road, Shanghai 20000 I, China \\ 2 Shanghai-Ministry Key Laboratory of Disease and Health Genomics, Chinese National Human Genome Center, Shanghai, China \\ ${ }^{3}$ Division of Rheumatology, Renji Hospital, Shanghai jiao-Tong University School of Medicine, Shanghai, China \\ ${ }^{4}$ Department of Surgery, University of Michigan, Ann Arbor, MI, 48109, USA \\ ${ }^{5}$ Department of Pathology and Medicine, The Warren Alpert Medical School of Brown University and Rhode Island Hospital, Providence, Rhode \\ Island, USA
}

\begin{abstract}
*Correspondence to: Jing-Yuan Fang or Jie Hong, Division of Gastroenterology and Hepatology, Renji Hospital, Shanghai Jiao-Tong University School of Medicine, Shanghai Institution of Digestive Disease; Key Laboratory of Gastroenterology and Hepatology, Ministry of Health; State Key Laboratory of Oncogene and Related Genes, 145 Middle Shandong Road, Shanghai 20000 I, China. e-mail: jingyuanfang@yahoo.com or jiehong97@gmail.com

Or Wei-biao Cao, Department of Pathology and Medicine, The Warren Alpert Medical School of Brown University and Rhode Island Hospital, 55 Claverick Street, Room 337, Providence, RI 02903, USA. e-mail: wcao@hotmail.com
\end{abstract}

†These authors contributed equally to this work.

\begin{abstract}
The polycomb group protein enhancer of zeste homologue 2 (EZH2), which has histone methyltransferase (HMT) activity, is overexpressed in malignant tumours. However, the role of EZH2 in colorectal cancer (CRC) invasion is little known. Here we investigated the clinical significance, biological effects, and mechanisms of EZH2 signalling. Knockdown of EZH2 significantly reduced cell invasion and secretion of matrix metalloproteinases 2/9 (MMP2/9) in in vitro studies. Knockdown of EZH2 dramatically increased overall survival and decreased metastasis of lung in in vivo studies. Conversely, overexpression of $E Z H 2$ significantly increased lung metastasis and shortened overall survival when compared with control tumours. EZH2-induced CRC cell invasion may depend on down-regulation of vitamin D receptor (VDR), which is considered to be a marker of CRC invasion. EZH2 regulates the histone trimethylation of lysine 27 (H3K27me3) in the VDR promoter. Moreover, we found that STAT3 directly binds to the EZH2 promoter and regulates VDR down-regulation in CRC cells. Significant inverse correlations were observed between the expression of EZH2 and PSTAT3 and that of VDR in CRC tissues compared with normal tissue in patients. We show the role of EZH2 in CRC metastasis and identify VDR as a target gene of EZH2. EZH2 expression may be directly regulated by STAT3, and STAT3 may play an important role in EZH2-mediated VDR down-regulation in CRC. This pathway may provide potential targets in aggressive CRC.

Copyright @ 2013 Pathological Society of Great Britain and Ireland. Published by John Wiley \& Sons, Ltd.
\end{abstract}

Keywords: colorectal cancer; invasion; EZH2; vitamin D receptor, STAT3

Received 22 November 2012; Revised 18 January 2013; Accepted 12 February 2013

No conflicts of interest were declared.

\section{Introduction}

Although colorectal cancer (CRC) mortality has decreased in the past decade, it remains a leading cause of morbidity and mortality [1,2]. Genetic and epigenetic alterations are important in the pathogenesis of CRC [3-5]. In addition to well-defined promoter DNA hypermethylation and histone deacetylation, deregulation of polycomb-induced gene silencing has been reported [6-8]. The enhancer of zeste homologue
2 (EZH2) protein is a core component of the polycomb repressive complex 2 (PRC2), which modifies transcription at the epigenetic level by affecting both histone and DNA methylation [9]. Recent studies have shown that EZH2 silences tumour suppressor gene expression, via the modification of lysine 27 in $\mathrm{H} 3$ histone tails in the promoter region of the target gene [10,11]. Overexpression of EZH2 correlates with invasiveness in several cancers $[12,13]$ but the role of $E Z H 2$ in the invasion of CRC remains unclear. 
As a member of the steroid hormone receptor superfamily, the vitamin $\mathrm{D}$ receptor (VDR) regulates gene expression in a ligand-dependent manner [14]. VDR was initially isolated from the nuclei of chicken small intestinal cells [15]. The active metabolite of vitamin $\mathrm{D}, 1 \alpha, 25$-dihydroxyvitamin $\mathrm{D} 3$, induces the expression of E-cadherin, which plays a causal role in the invasion of carcinoma cells that express the VDR [16,17]. Aggressive CRC cells with a high metastatic potential have low levels of VDR expression [18]. In addition, snail2 and snail1, the inducers of CRC cell invasion, may cooperate to repress expression of the VDR in CRC [19].Therefore, down-regulation of the VDR may be involved in the pathogenesis of $\mathrm{CRC}$ and could be a predictive marker of malignant potential [20].

Overexpression of $E Z H 2$ is associated with a poor outcome [21]. It has been reported that the mitogenactivated protein kinase (MAPK) pathway regulates EZH2 overexpression in subtypes of aggressive breast cancer [22]. The expression and function of EZH2 may be regulated by microRNA [23] and non-coding RNA [24]. However, the mechanism by which EZH2 is overexpressed in CRC remains unclear.

Accumulating evidence indicates that abnormalities in the Janus kinase/signal transducer and activator of transcription (JAK/STAT) pathway are involved in the oncogenesis of CRC $[25,26]$. As a key component of the JAK/STAT pathway, STAT3 is constitutively activated in CRC [27]. Yeh et al. postulated that EZH2 may be regulated by STAT3 in prostate cancer [28]. Furthermore, $\mathrm{EZH} 2$ has been shown to regulate $V D R$ gene expression in germinal centre B cells [29]. Whether EZH2 mediates VDR expression and the transcription factor(s) responsible for overexpression of EZH2 in CRC cells is not known. Our goal was to investigate this hypothesis.

\section{Materials and methods}

\section{Cell culture and treatment}

Human colon cancer cell lines HT29, SW1116, HCT116, and SW620 (ATCC, Manassas, VA, USA) were maintained in 1640 medium (Gibco, Carlsbad, CA, USA), respectively, supplemented with $10 \%$ fetal bovine serum (FBS). CRL-1790 cells, a 'normal' human colon epithelial cell line (ATCC), were also used. CRL-1790 cells were grown in F-12 medium enriched with $0.02 \mathrm{mg} / \mathrm{ml}$ insulin, $0.01 \mathrm{mg} / \mathrm{ml}$ transferrin, $25 \mathrm{nM}$ sodium selenite, $50 \mathrm{nM}$ hydrocortisone, $1 \mathrm{ng} / \mathrm{ml}$ epidermal growth factor, $0.01 \mathrm{mM}$ ethanolamine, $\quad 0.01 \mathrm{mM}$ phosphorylethanolamine, $100 \mathrm{pM}$ triiodothyronine, $0.5 \%(\mathrm{w} / \mathrm{v})$ bovine serum albumin, $10 \mathrm{mM}$ HEPES, $0.5 \mathrm{~mm}$ sodium pyruvate, and an extra $2 \mathrm{mM}$ L-glutamine (final concentration $4.5 \mathrm{mM}$ ). Cell density was maintained at approximately 100000 cells per millilitre of medium. Cells were then plated in fibronectin- and collagen type I-coated
T-25 flasks. All of the cells were incubated at $37^{\circ} \mathrm{C}$ in a humidified $5 \% \mathrm{CO}_{2}$ atmosphere.

\section{Immunohistochemical staining}

All specimens (tumour and parallel normal) were obtained from 129 patients with primary CRC, who underwent surgery at the Shanghai Renji Hospital from July 2009 to May 2012. All patients provided written informed consent before enrolment, and the study protocol was approved by the Ethics Committee of Shanghai Jiao-Tong University School of Medicine, Renji Hospital. The study was carried out according to the provisions of the Helsinki Declaration of 1975. None of the patients received pre-operative treatment. The expression levels of pSTAT3, STAT3, EZH2, and VDR were examined with primary antibodies (STAT3, dilution 1 : 100; pSTAT3, EZH2, and VDR, dilution $1: 50$ ) using the LSAB + kit (DakoCytomation, Copenhagen, Denmark) according to the manufacturer's instructions. Antibodies used in this study were purchased from Cell Signaling Technology Inc (Beverly, MA, USA).

The slides were examined independently by two investigators, who were blinded to the clinical and pathological data. Protein expression was quantified using a visual grading system based on the extent and intensity of staining (percentage of positive tumour cells was graded on a scale of 0-4: 0, none; 1, $1-25 \% ; 2,26-50 \% ; 3,51-75 \% ; 4,76-100 \%)$ and the intensity of staining (graded on a scale of $0-3$ : 0 , no staining; 1, weak staining; 2, moderate staining; 3 , strong staining). For further analysis, an index value was calculated as a product of the grades of the extent and intensity of staining to define the cut-off value for high expression of the proteins, and protein expression was classified into two categories: high (grades 4-12) and low (grades 0-3).

\section{Animal experiments}

We developed a CRC metastasis model in nude mice. HCT116 cells were infected with lenti-GFP-control virus to construct HCT116-GFP-control stable cell lines. HCT116 cells and HCT116-GFP-control stable cells $\left(2.5 \times 10^{6}\right)$ were suspended in $500 \mu$ l of PBS and injected through the tail veins of 6-week-old nude mice [30]. Forty days later, the NightOWL II LB 983 in vivo imaging system (Berthold Technologies, Bad Wildbad, Germany) was used to image the metastases. Preliminary data showed that GFP-positive subcutaneous metastasis was successfully detected after the injection of HCT116-GFP-control stable cells, but almost no GFP-positive subcutaneous metastasis was detected after the injection of PBS or HCT116 cells. All of the lentiviruses were provided by Shanghai Sunbio Biomedical Technology (Shanghai, China).

SW1116 cells were infected with lenti-GFP-EZH2 virus or lenti-GFP-control virus and HCT116 cells were infected with lenti-GFP-EZH2 shRNA virus or lenti-GFP-control shRNA virus to construct the 
SW1116-GFP-EZH2, SW1116-GFP-control, HCT116GFP-shEZH2, and HCT116-GFP-control shRNA stable cell lines, respectively. We injected PBS, SW1116 cells, SW1116-GFP-control cells, SW1116-GFP-EZH2 cells, HCT116 cells, HCT116-GFP-control shRNA cells or HCT116-GFP-shEZH2 cells into the tail veins of nude mice once a week. At 40 days, photographs were taken with the NightOWL II LB 983 imaging system. During this time, we recorded the survival time of the nude mice and survival curves were generated. The livers and lungs of the nude mice were excised post-mortem for haematoxylin and eosin staining [31]. Details of the CRC xenograft animal models may be found in the Supplementary methods. All experimental procedures were approved by the Institutional Animal Care and Use Committee.

\section{Chromatin immunoprecipitation (ChIP) and real-time ChIP}

ChIP assays were performed using the ChIP assay kit (Upstate, Charlottesville, VA, USA) according to the manufacturer's instructions. The protein-DNA complexes were precipitated using $5 \mu \mathrm{g}$ of antibodies against trimethyl-histone 3-Lys27 (3meH3K27; \#07-449, BD San Jose, CA, USA), STAT3 (\#9132, CST), EZH2 (\#4905, CST, San Jose, CA, USA) or IgG control (\#12-370, Santa Cruz, Dallas, TX, USA). Approximately 2-5 $\mu$ l of ChIP-enriched chromatin was subjected to a standard ChIP-PCR reaction, and the enrichment of specific genomic regions was assessed relative to either control IgG or control cells.

Real-time PCR was performed using an Applied Biosystems 7900 quantitative PCR system. Each PCR reaction was carried out in a $10 \mu \mathrm{l}$ reaction volume using $3 \mu \mathrm{l}$ of the eluted immunoprecipitated DNA. The amount of genomic DNA that co-precipitated with the specific antibody was calculated in comparison to the total input DNA used for each immunoprecipitation as follows: $\mathrm{CBTB}=\mathrm{CBTB}($ genomic input $)-\mathrm{CBTB}($ specific antibody), where $\mathrm{CBTB}$ (genomic input) and CBTB(specific antibody) are the mean threshold cycles of PCR performed in triplicate on DNA samples from the genomic input samples and the specific antibody samples, respectively. The ChIP primers for the VDR gene promoter were VDR-F (5'-CCCTTGGGTGAGATT- $\left.3^{\prime}\right)$ and VDR-R (5'-CTCCGCACGAATGG-3'). The ChIP primers for the $E Z H 2$ gene promoter were $E Z H 2-\mathrm{F}$ (5'-GGCGTCCTGTTGAA-3') and EZH2-R (5'CCGCCAACAAACTG-3'; Figure 6C). A ChIP primer pair for $G A P D H$ was used as a negative control for STAT3 binding: (5'-CACCGTGTGCCCAAGACCTC$\left.3^{\prime}\right)$ and (5'-CAGCCCTGTAGCCTGGACCT-3').

\section{Statistical analysis}

Statistical analysis was performed with SPSS 13.0 software (SPSS Inc, Chicago, IL, USA). For clinicopathological analysis, the chi-squared test or Fisher's exact test (two-sided) was performed. The Kaplan-Meier method was used to estimate the overall survival, and the log-rank test was used to evaluate the differences between survival curves. Correlation analyses were performed for the expression of pSTAT3, STAT3, $E Z H 2$, and VDR. Statistical tests and $p$ values were two-sided. Results were considered significant if the $p$ value was less than 0.05 .

\section{Results}

\section{Expression of EZH2 in CRC tissues and cells}

As shown in Figure 1A, EZH2 staining was mainly detected in the nuclei of cells in CRC tissue samples, with almost no $E Z H 2$ staining observed in normal epithelial tissues. Furthermore, the expression of EZH2 was much higher in tissues from CRC patients with metastasis than in those without metastasis. We found an association between $\mathrm{EZH} 2$ expression and several known clinicopathological features in CRC. EZH2 expression was positively correlated with location, TNM stage, histological grade, vascular invasion, and lymph node metastasis (Supplementary Table 1). Western blot analysis (Figure 1B) showed that EZH2 was not detectable in normal human colon epithelial cells (CRL-1790), but EZH2 expression was significantly increased in all of the CRC cell lines tested, especially in highly invasive CRC cells.

\section{EZH2 plays an important role in CRC cell invasion}

EZH2 may mediate cell proliferation and invasion in human cancers $[8,32,33]$. To study this in CRC, first, we introduced lenti-EZH2 shRNA-1/2 viruses or a lenti-EZH2 overexpression virus into CRC cells. Lenti-EZH2 shRNA-1/2 viruses successfully decreased EZH2 expression, respectively (Supplementary Figure 1A). Moreover, introduction of the lenti-EZH2 overexpression virus remarkably increased EZH2 expression (Supplementary Figure 1B). There was no significant change of $E Z H 2$ expression after transduction of the control shRNA or control viruses when compared with control CRC cells.

In Transwell cell invasion assays, representative data showed that knockdown of EZH2 expression significantly reduced the invasion ability of $\mathrm{CRC}$ cells (Figure 1C). Moreover, overexpression of EZH2 dramatically increased the invasion ability of CRC cells (Figure 1C), indicating that EZH2 may have a significant effect on cell migration and invasion in CRC cells. In wound healing assays, less wound closure was observed in CRC cells with knockdown of EZH2 compared with control. Furthermore, more wound closure was observed in CRC cells with overexpression of EZH2 compared with control (Figure 1D). This result was further confirmed by ELISA assays, as knockdown of $E Z H 2$ significantly reduced the secretion of MMP2 and MMP9 in CRC cells (Supplementary Figure 2). 


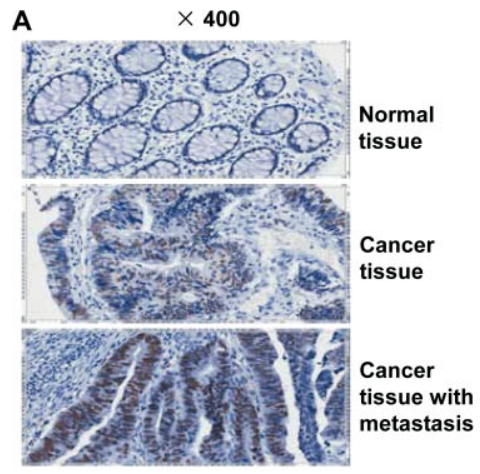

B CRL-1790 HT29 SW1116 HCT116 SW480 SW620
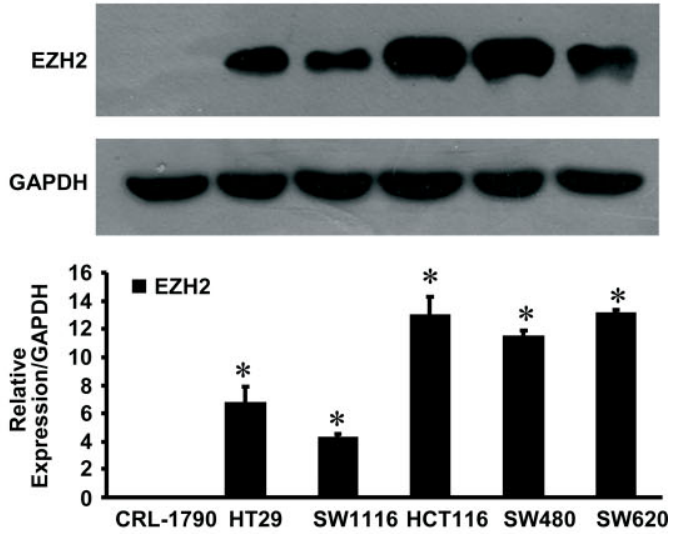

C
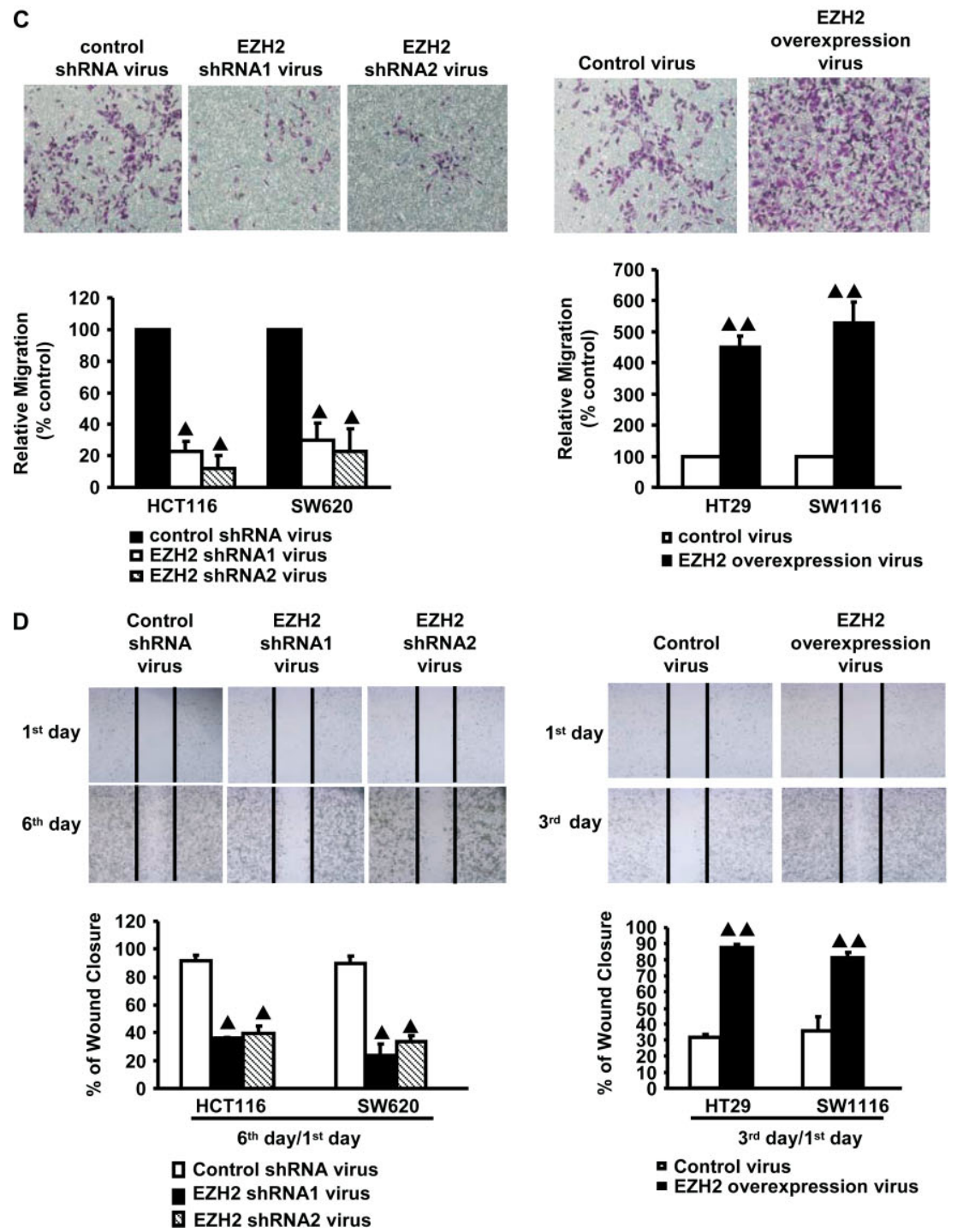

Figure 1. Expression and function of EZH2 in CRC tissues and cells. (A) Immunohistochemical analysis of EZH2 in normal colorectal mucosa, CRC tissues, and CRC tissues from patients with metastasis (original magnification $\times 400$ ). (B) Western blot analysis showing almost no expression of EZH2 in a normal human colon epithelial cell line, CRL-1790; however, the expression of EZH2 was significantly increased in all CRC cell lines, especially in highly invasive CRC cells. (C) Transwell Matrigel invasion assays were performed in CRC cells infected with control shRNA or EZH2 shRNA viruses or control or EZH2 overexpression virus. Cells were observed under a light microscope and photographed. Cells were counted from five random microscopic fields $(200 \times)$ per insert in triplicate. The migrated cell numbers were normalized to that of the control group. Data are shown as mean \pm SD from three separate experiments. (D) Wound healing assays were performed in CRC cells after infection with the $E Z H 2$ overexpression virus, control virus, control shRNA virus or $E Z H 2$ shRNA virus. $n=3$, $\mathrm{ANOVA}^{*}{ }^{*} p<0.05$, compared with CRL-1790 cells; $n=3$, ANOVA, $\boldsymbol{\Delta} p<0.05$, compared with the control shRNA virus; $n=3, \boldsymbol{\Delta} \mathbf{\Delta} p<0.05$ (Student's $t$ test). 
We then explored the functional impact of $E Z H 2$ overexpression/knockdown. Different stable cells were transplanted into BALB/c-nu/nu mice via tail vein injection. Increased numbers of subcutaneous metastases were detected after injection of SW1116-GFPEZH2 cells, compared with mice injected with control cells (Figure 2A). Mice inoculated with SW1116GFP-EZH2 cells had a shorter overall survival time (mean 65.7 days) compared with those injected with control cells (mean 76.4 days; Figure 2C). Haematoxylin and eosin staining showed that fewer metastatic CRC cells were observed in the lungs of nude mice at 17 weeks after injection of SW1116-GFP-control cells, while most of the mice injected with SW1116GFP-EZH2 cells displayed significant lung metastases (Figure 2E and Supplementary Table 2). Fewer subcutaneous metastases were detected after down-regulation of EZH2 (Figure 2B). Knockdown of EZH2 remarkably increased the overall survival time and significantly decreased lung metastasis of tumour cells in vivo (Figures 2D, 2E, and Supplementary Table 2). These data suggest that $E Z H 2$ expression plays a critical role in CRC cell motility and metastasis.

We compared the survival time in patients with $E Z H 2$-expressing tumours $(n=17)$ with that of patients with $E Z H 2$-negative tumours ( $n=16$; Figure $2 \mathrm{~F}$ ). The cumulative survival rate was significantly better in patients with $E Z H 2$-negative tumours than in those with $E Z H 2$-positive tumours $(p<0.05, H R=0.1973$, 95\% CI 0.06-0.66), indicating that EZH2 overexpression is significantly associated with patient survival in CRC.

\section{EZH2 negatively regulates VDR expression in CRC cells and $E Z H 2$-induced CRC cell invasion depends on the repressing of VDR}

E-cadherin expression is regulated by $E Z H 2$ via the histone methylation of E-cadherin promoter [34,35]. Velichutina et al. predicted that $V D R$ is a target of $E Z H 2$ [29]. We examined whether $E Z H 2$ regulates VDR expression in CRC cells. Real-time PCR data showed that up-regulation of EZH2 significantly down-regulated VDR expression in SW1116 cells (Figures 3A and 3C). Conversely, down-regulation of $\mathrm{EZH} 2$ significantly increased the mRNA level (Figure 3B) and protein levels (Figure 3D) of VDR in HCT116 cells. The data suggest that EZH2 may regulate VDR expression in CRC cells. In addition, overexpression of VDR significantly decreased the invasion ability of CRC cells under basal conditions. Upregulation of VDR blocked $E Z H 2$-induced cell invasion in SW1116 cells (Figure 3E) and down-regulation of VDR restored the EZH2 shRNA-induced decrease in cell invasion ability in HCT116 cells (Figure 3F), indicating that $\mathrm{EZH} 2$-induced CRC cell invasion may depend on the suppression of VDR expression.

To explore the mechanism of EZH2 -induced VDR down-regulation in CRC cells, we constructed a luciferase reporter plasmid containing part of the $V D R$ promoter region. Knockdown of EZH2 significantly increased the transcriptional activity of the VDR promoter in HCT116 cells (Figure 4A), indicating that EZH2 may mediate VDR expression by regulating the transcriptional activity of the $V D R$ promoter. We then examined the H3K27 methylation level of the $V D R$ promoter after knockdown of EZH2. As shown in Figure 4B, VDR genomic DNA was detectable in a ChIP assay when either an H3K27me3 antibody or EZH2 antibody was used, and Figure 4C shows that knockdown of $E Z H 2$ significantly decreased the $\mathrm{H} 3 \mathrm{~K} 27$ histone methylation level of the $V D R$ promoter, suggesting that $E Z H 2$ may regulate the expression of VDR by modulating the $\mathrm{H} 3 \mathrm{~K} 27$ histone trimethylation in the $V D R$ promoter region.

Supplementary Tables 1 and 4 show the frequencies of EZH2 and VDR expression in 129 paired samples of normal and CRC tissues from patients undergoing colorectal surgery. In consecutive tissue sections (Figure 4D), EZH2 was expressed at higher levels in the CRC samples than in the control tissues. In contrast, VDR was expressed at lower levels in the tumour tissues than in the normal colon epithelial samples. Our data suggest that $E Z H 2$ is significantly up-regulated in CRC (chi-squared test, $p<0.0001$ ), while VDR is significantly down-regulated (chi-squared test, $p=0.012$ ). Additionally, the expression levels of EZH2 and VDR were inversely correlated (Spearman's correlation coefficient $r=-0.65720 ; p<0.0001)$.

\section{STAT3 regulates $E Z H 2$ expression in CRC cells}

STAT3 mediates the invasion of cancer cells [36,37]. Since EZH2 mediates CRC cell invasion and EZH2induced CRC cell invasion depends on the suppression of VDR expression, we next determined whether STAT3 regulates the expression of EZH2 and VDR in CRC cells. Western blot analysis showed that knockdown of STAT3 significantly decreased EZH2 expression and increased VDR expression in CRC cells (Figures 5A and 5B). Moreover, EZH2 expression was markedly increased and VDR expression was significantly decreased after overexpression of STAT3 in CRC cells (Figures 5C and 5D), suggesting that STAT3 may play an important role in $E Z H 2$ up-regulation and VDR down-regulation. Knockdown of EZH2 blocked STAT3-induced down-regulation of VDR expression levels in CRC cells (Figures $5 \mathrm{E}$ and $5 \mathrm{~F}$ ), indicating that STAT3-mediated VDR down-regulation depends on the activation of $E Z H 2$.

To explore the mechanism of STAT3-induced EZH2 up-regulation in CRC cells and based on our preliminary luciferase reporter gene data (Supplementary Figure 3), we constructed a luciferase reporter plasmid containing part of the EZH2 promoter region (from -420 to $+80 \mathrm{nt}$ ). Overexpression of STAT3 dramatically increased the transcriptional activity of the EZH2 promoter in HCT116 cells (Figure 6A). Moreover, DNA sequence analysis of the EZH2 promoter region revealed six putative STAT3 binding sites. The 
A
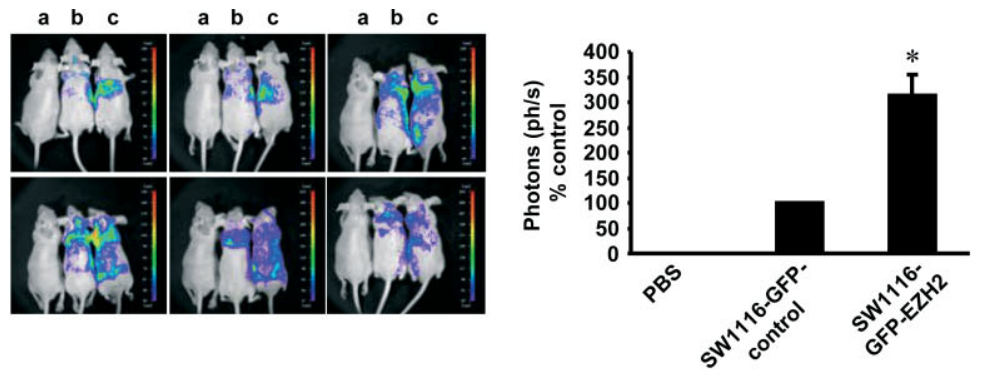

B
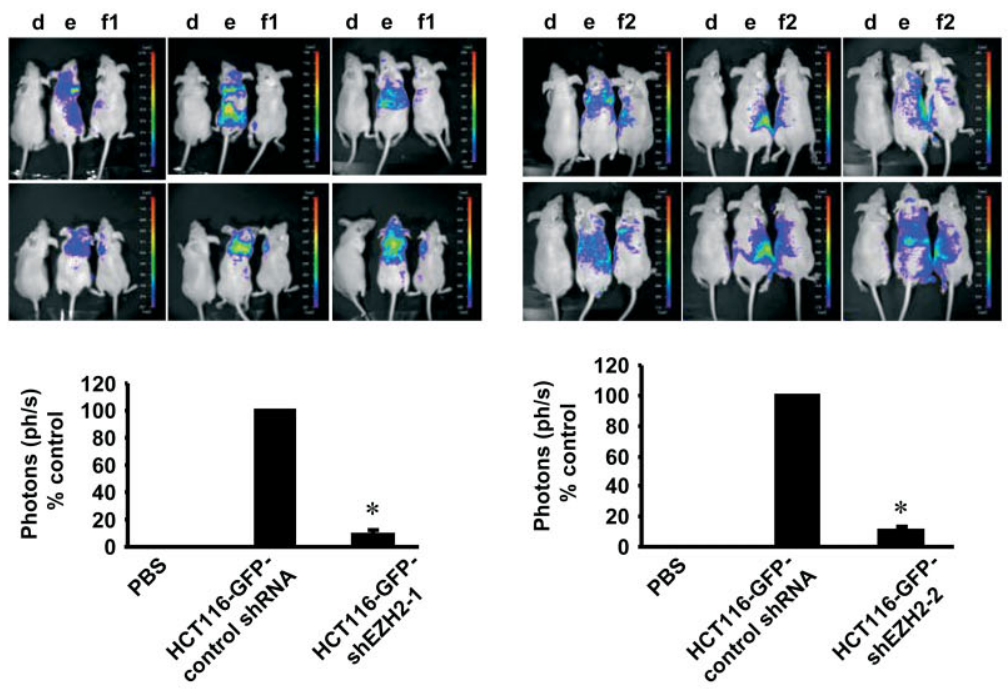

C Survival of Two groups:
Survival proportions ... SW1116-GFP-control

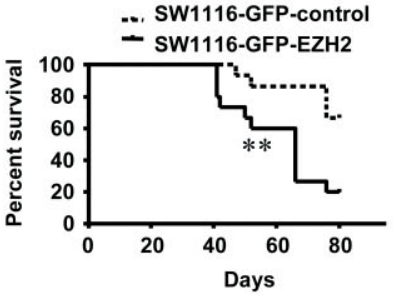

E

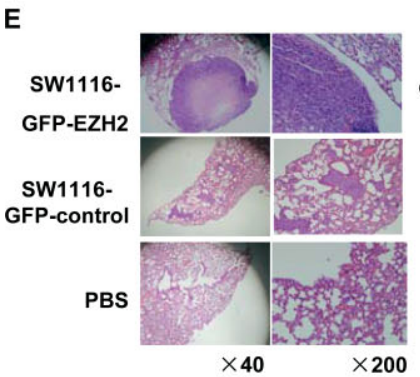

D Survival of Two groups: Survival proportions - HCT116-GFP-control shRNA $\therefore$ HCT116-GFP-shEZH2-1

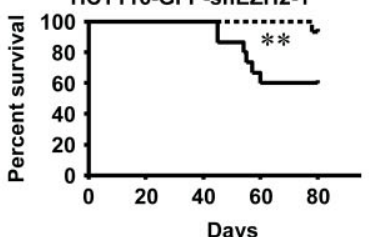

Days

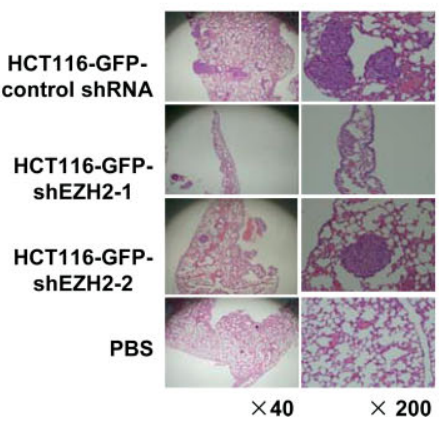

Survival of Two groups: Survival proportions

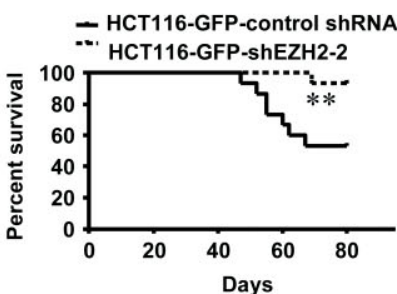

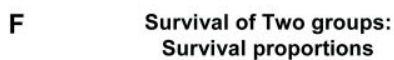

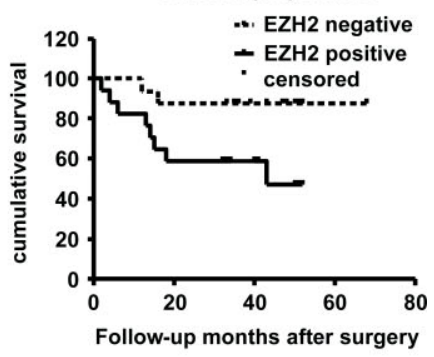

Figure 2. Functional impact of EZH2 overexpression/knockdown on the metastatic potential of CRC cells in vivo. (A) Assessment of the subcutaneous metastatic capacity of $2.5 \times 10^{6}$ SW1116-GFP-control or SW1116-GFP-EZH2 cells after inoculating nude mice via tail vein injection. Biofluorescence images and summarized data show subcutaneous metastasis and total photon flux for each treatment group (a: mice injected with PBS; b: mice injected with SW1116-GFP-control stable cells; c: mice injected with SW1116-GFP-EZH2 stable cells). $n=15$, ANOVA, ${ }^{*} p<0.0001$. (B) Assessment of subcutaneous metastatic capacity of $2.5 \times 10^{6}$ HCT116-GFP-control shRNA or HCT116-GFP-shEZH2-1/2 tumour cells by inoculating nude mice via tail vein injection. Biofluorescence images and summarized data show subcutaneous metastasis and total photon flux for each treatment group (d: mice injected with PBS; e: mice injected with HCT116-GFP-control shRNA stable cells; f1: mice injected with HCT116-GFP-shEZH2-1 stable cells; f2: HCT116-GFP-shEZH2-2 stable cells). $n=15$, ANOVA, ${ }^{*} p<0.0001$. (C, D) Survival curves for mice. $n=15^{* *} p<0.05$ (Student's $t$-test), compared with SW1116-GFP-control cells or HCT116-GFP-control shRNA cells. (E) Increased numbers of metastatic CRC cells were detected in the lungs of nude mice at 17 weeks after the injection of the SW1116-GFP-EZH2 group compared with the SW1116-GFP-control group. Fewer metastatic CRC cells were detected in the lungs of nude mice injected with HCT116-GFP-shEZH2-1/2 cells at 17 weeks compared with mice injected with HCT116-GFP control shRNA cells.(F) Survival analysis showed that EZH2-positive tumours have an unfavourable prognosis compared with EZH2-negative tumours in patients $(p<0.05, \mathrm{HR}=0.1973,95 \% \mathrm{Cl} 0.06-0.66)$. 
A SW1116 cells

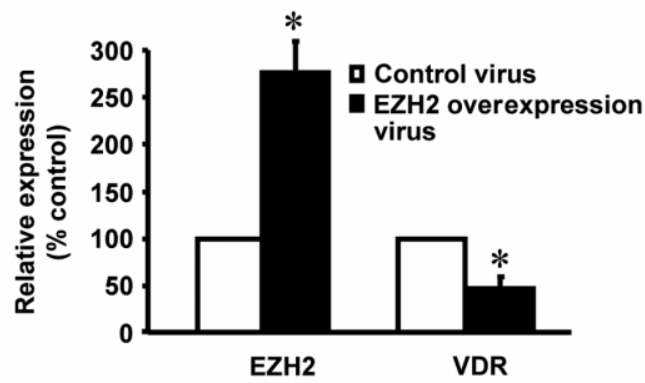

C SW1116 cells
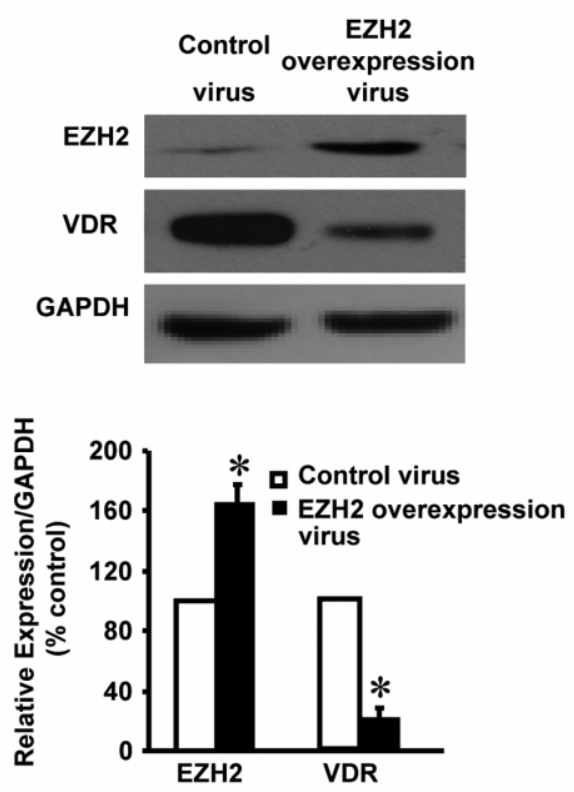

E SW1116 cells

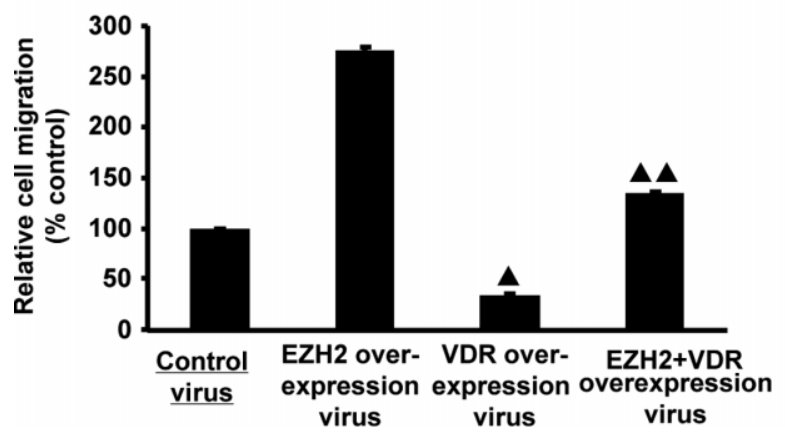

B HCT116 cells

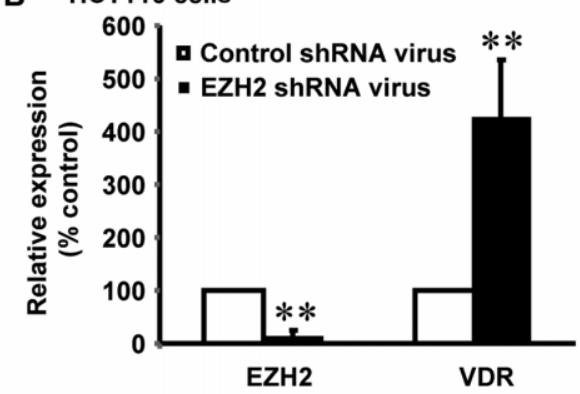

D HCT116 cells
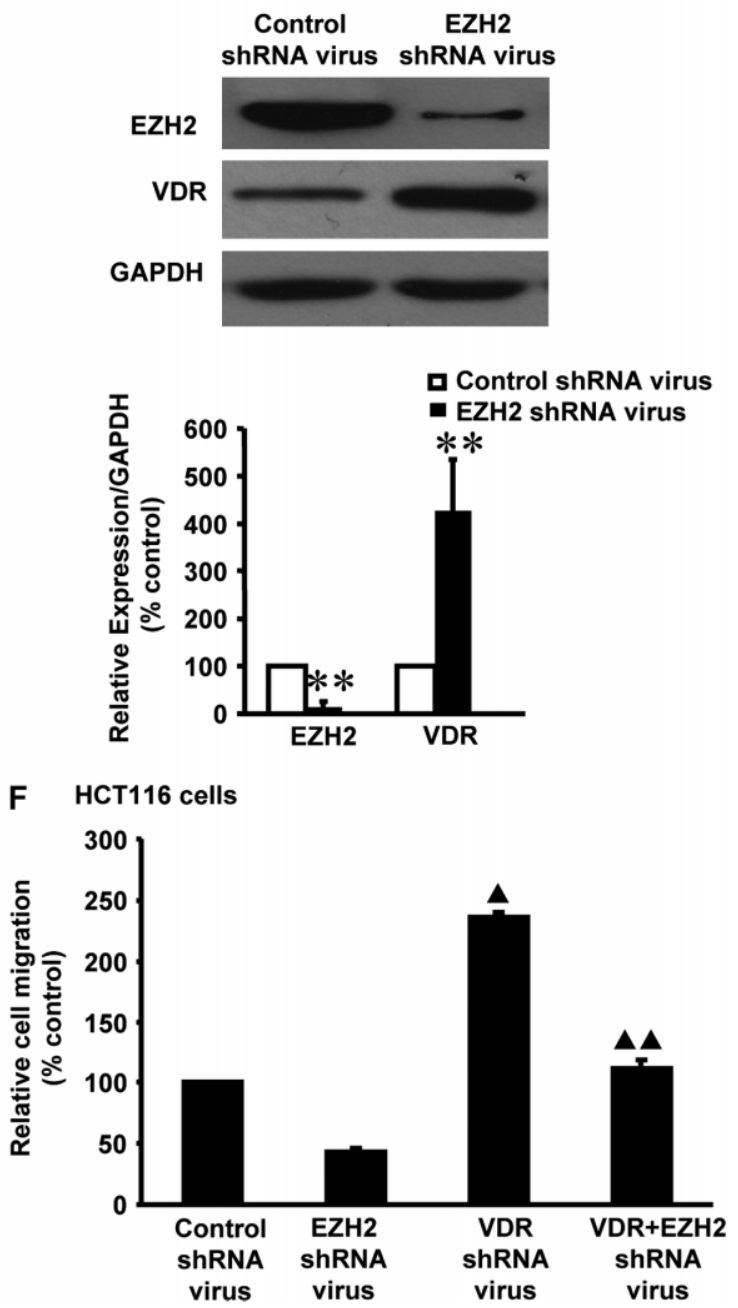

Figure 3. Role of EZH2 in VDR regulation and effect of VDR on EZH2-induced CRC cell invasion. (A) Real-time PCR data show that infection of EZH2 overexpression virus dramatically increased EZH2 mRNA expression and decreased VDR mRNA expression in SW1116 cells. $n=3,{ }^{*} p<0.01$ (Student's $t$-test). (B) Real-time PCR show that EZH2 shRNA virus significantly decreased the mRNA level of the EZH2 gene and increased the mRNA level of the VDR gene in HCT116 CRC cells. $n=3,{ }^{* *} p<0.01$ (Student's $t$-test). (C)Western blot analysis data show that overexpression of EZH2 significantly decreased the expression of VDR in SW1116 cells. $n=3,{ }^{*} p<0.01$ (Student's $t$-test). (D) Knockdown of EZH2 dramatically increased the expression of VDR in HCT116 cells. $n=3,{ }^{* *} p<0.01$ (Student's $t$-test). (E, F) Transwell Matrigel invasion assays were performed in CRC cells infected with different viruses as indicated. Cells were observed under a light microscope and photographed. Cells were counted from five random microscopic fields $(200 \times)$ per insert in triplicate. The migrated cell numbers were normalized to that of the control group. Data are shown as mean \pm SD from three separate experiments. $n=3$, ANOVA, $p<0.01$, compared with control; $p<0.05$, compared with EZH2 overexpression virus or EZH2 shRNA virus. 
A HCT116 cells

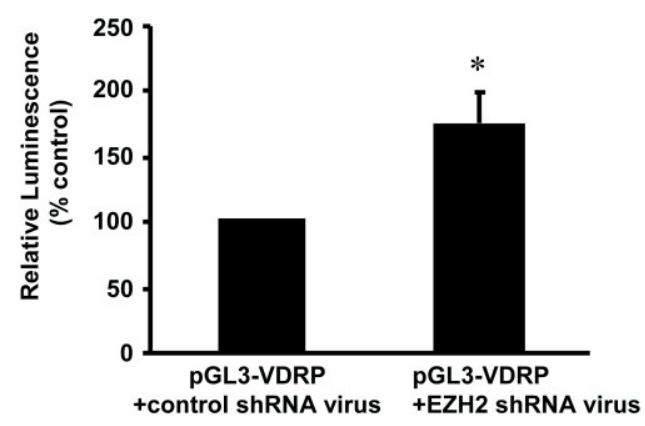

C HCT116 cells

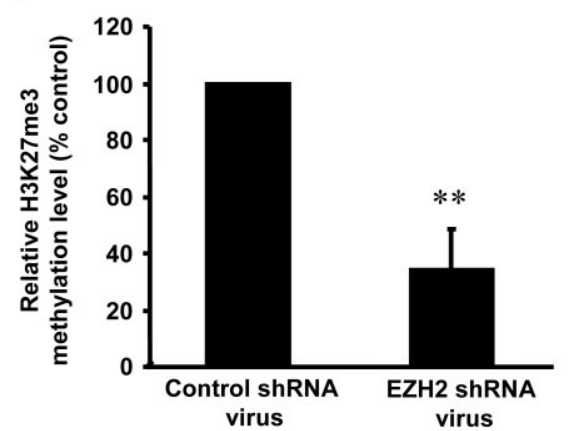

D

B HCT116 cells
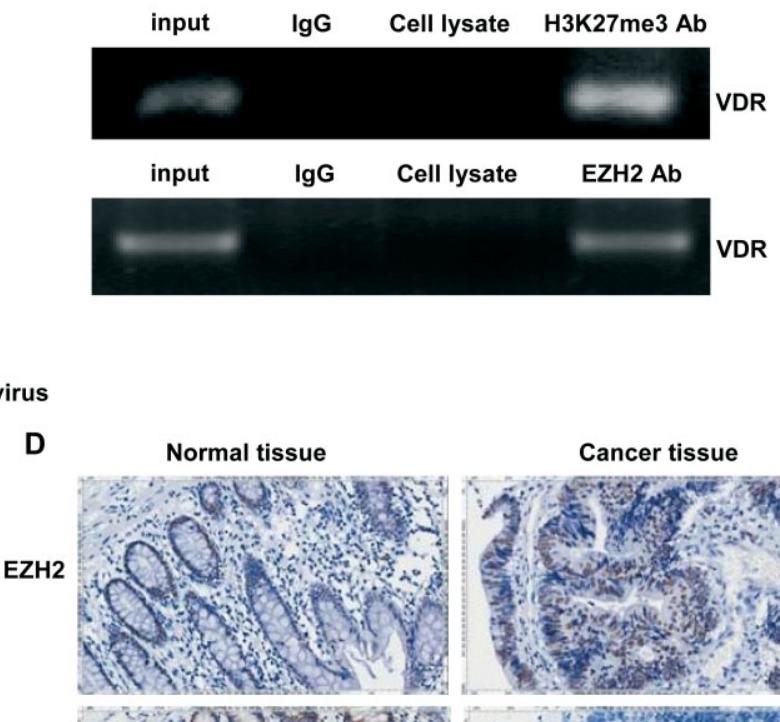

Normal tissue

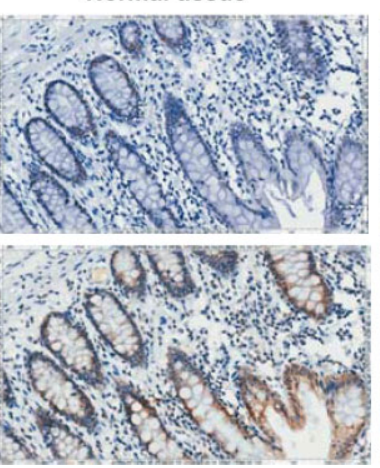

Cancer tissue

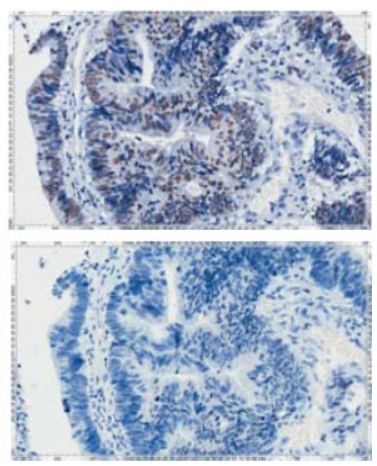

Figure 4. The mechanism of EZH2-mediated VDR down-regulation in CRC cells. (A) Knockdown of EZH2 significantly increased the luciferase activity of the VDR gene promoter. $n=3,{ }^{*} p=0.0005$ (Student's t-test). (B) The band in the gel of the ChIP assay suggests that the H3K27me3 site exists in the VDR gene promoter. Input DNA was used as a positive control; cell lysates incubated with non-relevant rabbit IgG or cell lysate without antibody incubation were used as negative controls. (C) Real-time PCR of ChIP samples showed that knockdown of EZH2 dramatically decreased the trimethylation level of H3K27 in the VDR promoter in HCT116 cells. $n=3,{ }^{* *} p=0.0097$ (Student's t-test). (D) Immunohistochemical analysis of consecutive human CRC tissue sections showed that higher levels of EZH2 nuclear staining were observed in CRC samples than in normal colonic epithelial samples, and the expression of VDR showed the opposite trend (original magnification $\times 400$ ).

transcriptional activity of the EZH2 promoter was significantly decreased after mutation of STAT3 binding sites in the $E Z H 2$ promoter (Figure 6B). The data suggest that STAT3 may activate the EZH2 promoter. In a ChIP assay, we used a pair of primers covering a region from -141 to $48 \mathrm{nt}$, containing four putative STAT3 binding sites, in the EZH2 promoter. Figure 6D shows that $E Z H 2$ genomic DNA was detectable in the ChIP assay with STAT3 antibody incubated. ChIP realtime PCR analysis demonstrated that overexpression of STAT3 significantly increased the recruitment of STAT3 to the EZH2 promoter (Figure 6E). The data indicate that STAT3 may bind to the EZH2 promoter. In addition, down-regulation of EZH2 blocked the STAT3-induced increase in cell invasion in HCT116 cells (Figure 6F), indicating that EZH2 may participate in STAT3-induced CRC cell invasion.

\section{Expression of pSTAT3, STAT3, EZH2, and VDR in CRC tissues}

We also found an association between pSTAT3/VDR expression and several known clinicopathological features in CRC (Supplementary Tables 3 and 4). The results suggest that pSTAT3 expression was positively correlated with histological grade, vascular invasion, and lymph node metastasis, and VDR expression was negatively correlated with TNM stage, vascular invasion, and lymph node metastasis. Furthermore, we used univariate and multivariate analyses to analyse whether the expression of EZH2, VDR, and pSTAT3 was associated with metastasis (vascular invasion and lymph node metastasis) in human CRC (Table 1). In univariate analysis, EZH2, VDR, and pSTAT3 were significantly associated with vascular invasion and lymph node metastasis, respectively; however, only $E Z H 2$ was found to be significantly associated with vascular invasion and lymph node metastasis in CRC patients in multivariate analyses.

We found that $85.5 \%$ (71/83) of the CRC patients who had low levels of VDR expression in their tumour tissues exhibited up-regulation of EZH2 $(p<0.001)$. The tumour tissues of $83.33 \%(65 / 78)$ of the patients displaying high levels of pSTAT3 also expressed high levels of EZH2 $(p<0.001)$. Furthermore, $81.93 \%$ $(68 / 83)$ of the patients with low levels of VDR in their tumour tissues exhibited up-regulation of pSTAT3 $(p<0.001)$ (Supplementary Table 5).

Supplementary Table 6 shows the frequencies of STAT3, pSTAT3, EZH2, and VDR expression levels in tumour tissues formed by CRC cells in which STAT3 was down-regulated or up-regulated, compared with control tumour tissues, in the nude mouse xenograft model. High levels of pSTAT3 were found 
A SW1116 cells
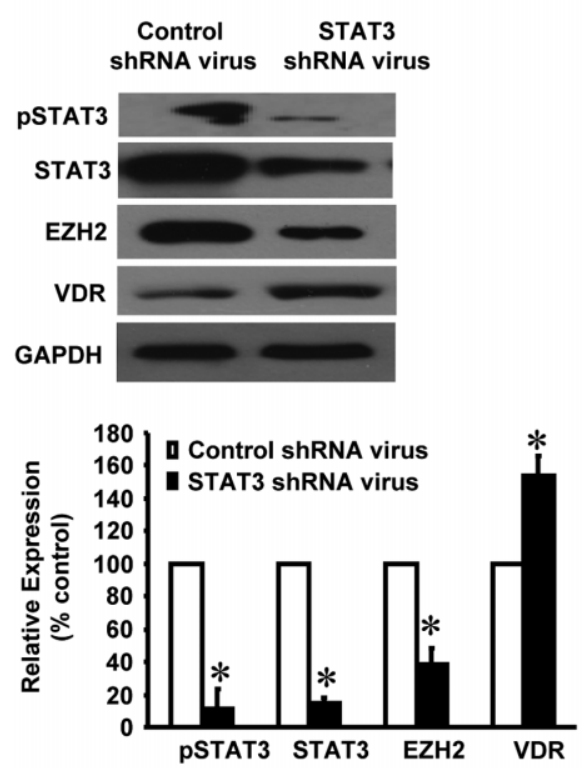

$\begin{array}{cc}\text { SW1116 cells } \\ \begin{array}{c}\text { Control } \\ \text { virus }\end{array} & \begin{array}{c}\text { STAT3 } \\ \text { Overexpression } \\ \text { virus }\end{array}\end{array}$
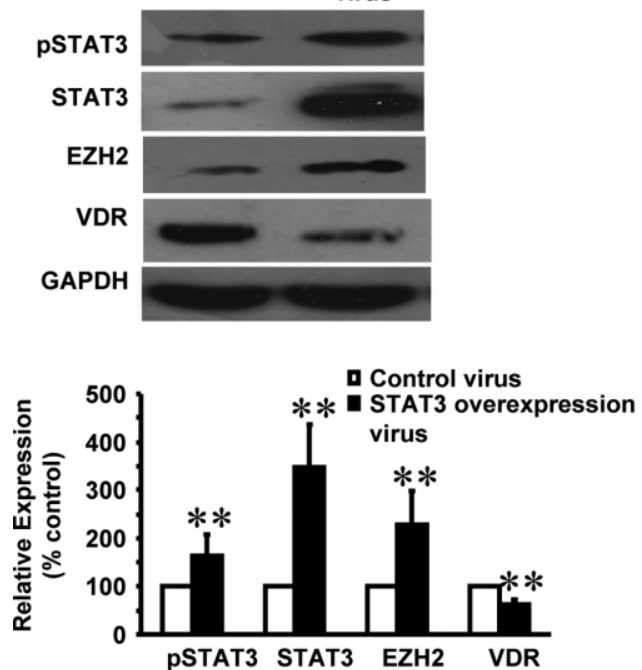

E HCT116 cells

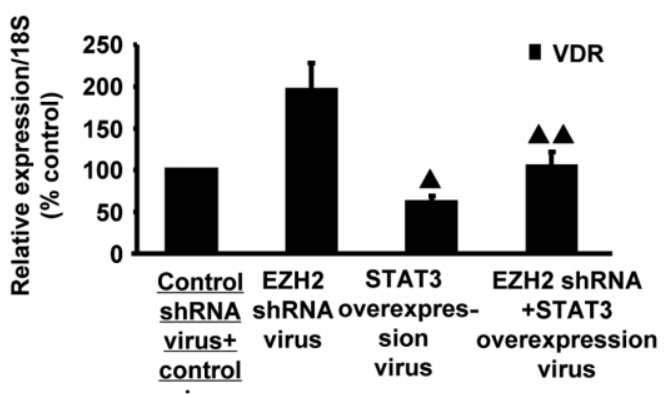

B

HCT116 cells

Control STAT3

ShRNA virus ShRNA virus
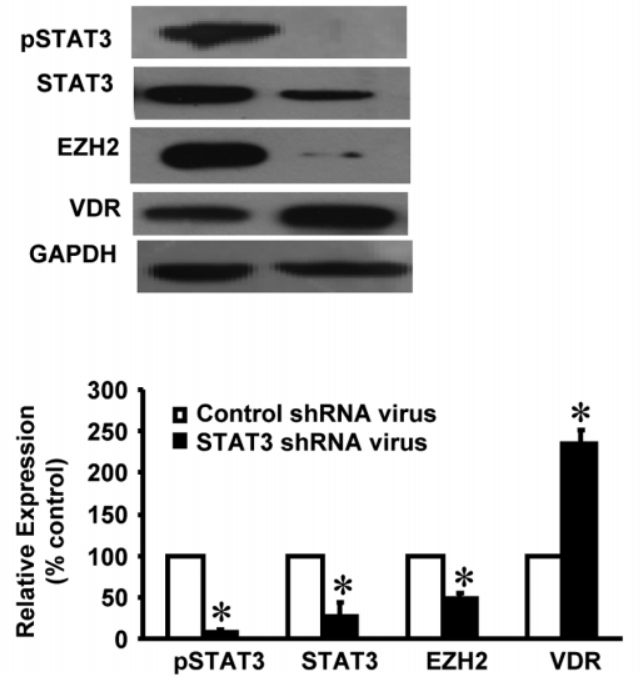

D
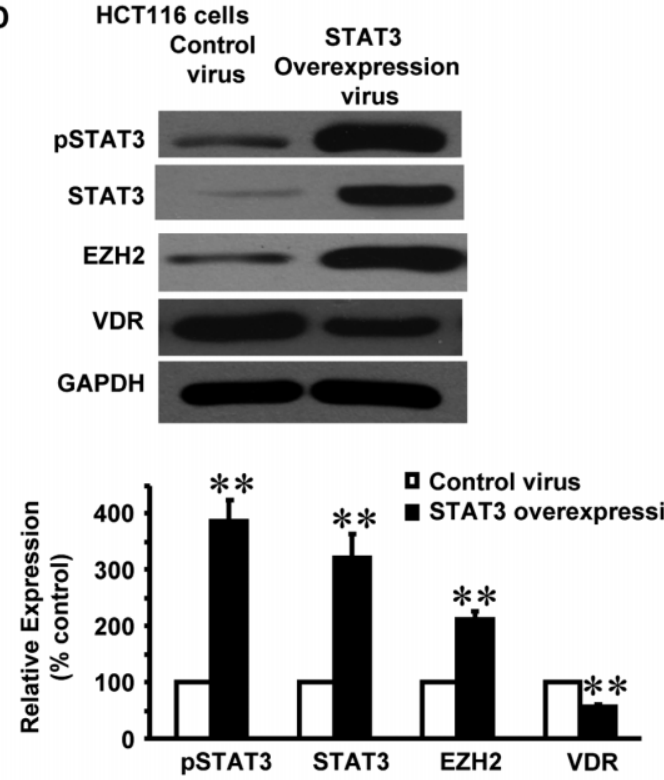

F HCT116 cells

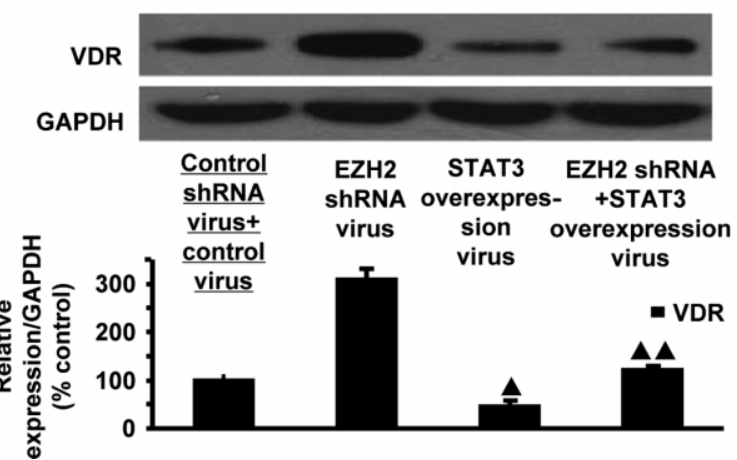

Figure 5. Effect of STAT3 on EZH2 and VDR expression in CRC cells. Western blot analysis data show that infection of STAT3 shRNA virus dramatically inhibited the phosphorylation and expression of STAT3. STAT3 down-regulation significantly decreased the expression of EZH2 and increased the expression of VDR in SW1116 (A) and HCT116 cells (B). ${ }^{*} p<0.01$ (Student's t-test), compared with control shRNA virus. Western blot analysis data show that the phosphorylation and expression of STAT3 were successfully increased after introducing STAT3 overexpression virus. EZH2 expression was significantly increased and VDR expression was decreased after the overexpression of STAT3 in SW1116 (C) and HCT116 cells (D). $n=3,{ }^{* *} p<0.01$ (Student's t-test), compared with control virus. Real-time PCR and western blot data show that introducing EZH2 shRNA virus significantly blocked STAT3-mediated VDR down-regulation in CRC cells (E, F). ANOVA, $p<0.05$, compared with control virus group; $p<0.05$, compared with STAT3 overexpression virus group. 


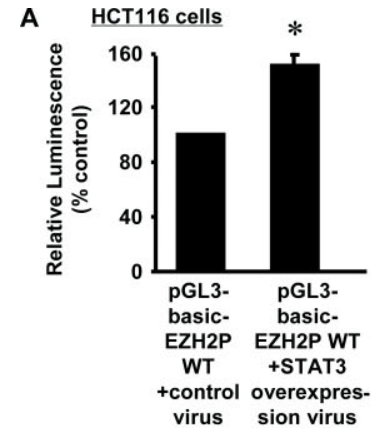

C

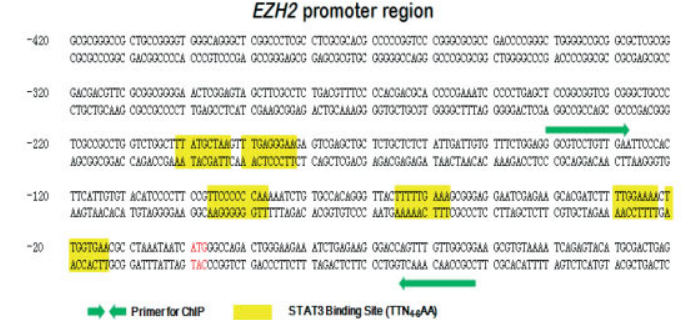

B

WT

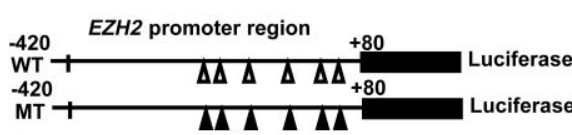

$\Delta$ WT STAT3 binding sites $\boldsymbol{\Lambda}$ MT STAT3 binding sites MT

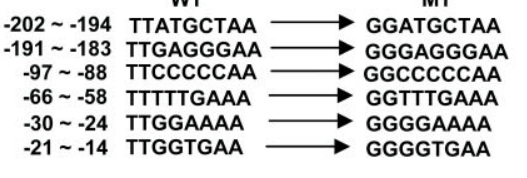

D HCT116 cells

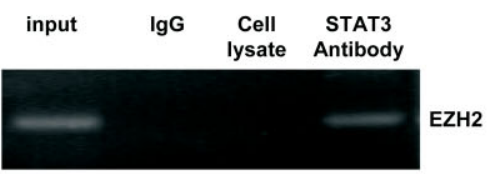

E

HCT116 cells

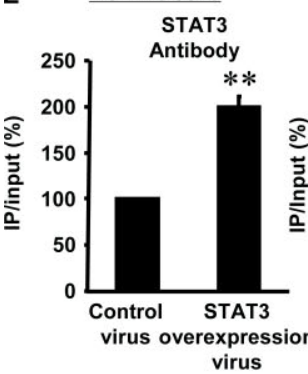
STAT3
Antibody
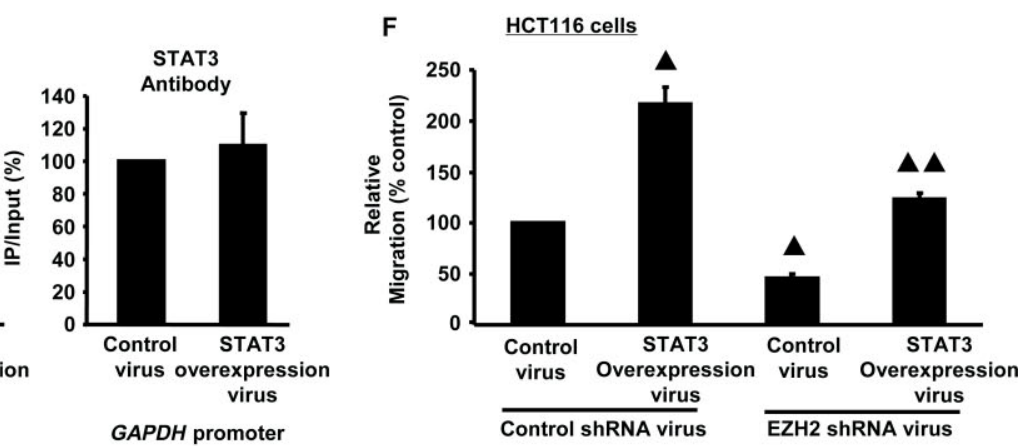

G

EZH2 promoter

GAPDH promoter

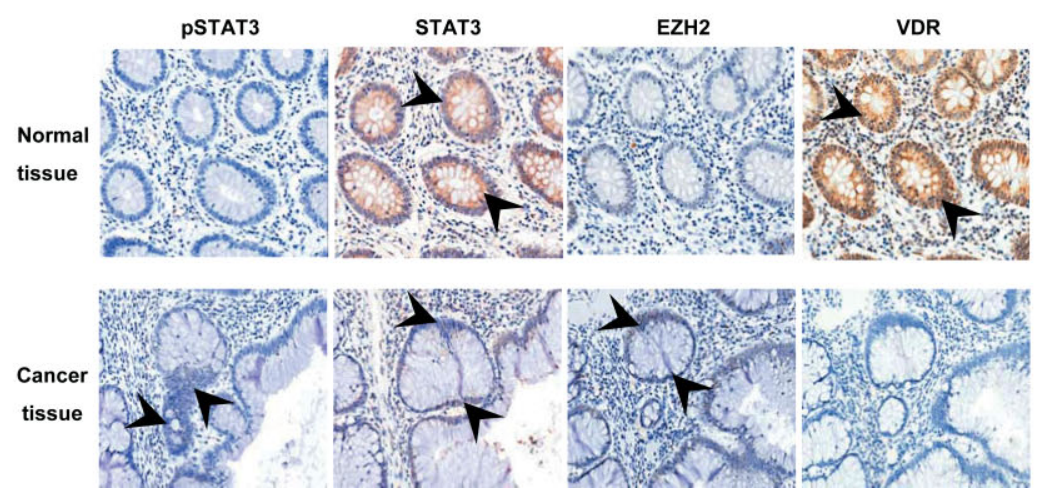

Figure 6. The mechanism of STAT3-mediated EZH2 up-regulation in CRC cells. (A) STAT3 up-regulation dramatically increased the luciferase activity of the EZH2 gene promoter, suggesting that STAT3 regulates the expression of EZH2 by modulating the transcriptional activity of the EZH2 gene. $n=3,{ }^{*} p<0.01$ (Student's $t$-test). (B) STAT3 binding sites in the EZH2 gene promoter. White and black triangles indicate a wild-type or mutant sequence for STAT3 binding sites, respectively. WT = wild type; MT = mutant type of each STAT3 mutation binding site. Mutation of STAT3 binding sites significantly decreased the transcriptional activity of the EZH2 promoter in the luciferase assay. $n=$ $3,{ }^{*} p<0.01$ (Student's $t$-test). (C) Bioinformatic analysis of STAT3 transcriptional factor binding sites in part of the EZH2 gene promoter region. Numbers on the left-hand side indicate the locations upstream of the first base of the initial translation site. STAT3 binding sites are highlighted, and the DNA sequence encompassed by two arrows was amplified in the ChIP assay. EZH2 DNA was detected in the chromatin sample immunoprecipitated from HCT116 cells using an antibody against STAT3 (D), suggesting that STAT3 binds to the EZH2 promoter. (E) Real-time PCR of the ChIP samples showed that overexpression of STAT3 dramatically increased the binding efficiency of STAT3 to the EZH2 promoter in CRC cells. ChIP assay real-time PCR was performed using GAPDH as a negative control for STAT3 binding. $n=3,{ }^{* *} p<0.01$ (Student's $t$-test). (F) Transwell Matrigel invasion assays were performed in CRC cells infected with different viruses as indicated. Cells were observed under a light microscope and photographed. Cells were counted from five random microscopic fields $(200 \times)$ per insert in triplicate. The migrated cell numbers were normalized to that of the control group. Data are shown as mean \pm SD of three separate experiments. $n=3$, ANOVA, $p<0.01$, compared with control virus plus control shRNA virus; $p<0.05$, compared with STAT3 overexpression virus plus control shRNA virus. (G) Immunohistochemical analysis for pSTAT3 and EZH2 in consecutive human CRC tissue sections. EZH2 and PSTAT3 were expressed at higher levels in CRC than in normal colonic epithelial samples, and the expression of VDR showed the opposite trend. There was no significant difference in STAT3 expression in normal colonic epithelial samples or CRC tissues (original magnification $\times 400$ ). 
Table 1. Univariate and multivariate analyses using the Cox proportional hazards model. EZH2 expression is significantly associated with the metastasis (vascular invasion and lymph node metastasis) of patients

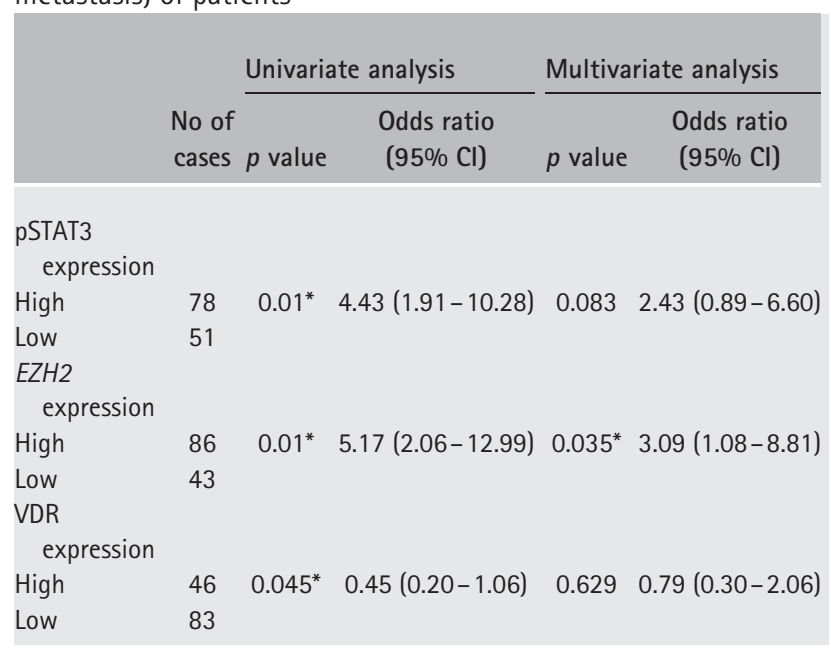

$\mathrm{Cl}=$ confidence intercal.

*Statistically significant.

in STAT3-overexpressing tumours, and there was almost no pSTAT3 staining in the STAT3-knockdown tumours (Figure 7A). High levels of EZH2 expression were found in $87 \%$ of the STAT3-overexpressing tumours and $7 \%$ of the STAT3-knockdown tumours (Figure 7C). VDR was highly expressed in 79\% of the STAT3-knockdown tumours, and no VDR staining was detected in the STAT3-overexpressing tumours (Figure 7D). Our data suggest that the expression levels of pSTAT3 and EZH2 were significantly upregulated (chi-squared test, $p<0.001$ ), while VDR expression was significantly decreased (chi-squared test, $p<0.001)$ in STAT3-overexpressing tumours. Conversely, the expression of pSTAT3 and EZH2 was significantly decreased (chi-squared test, $p<0.001$ ), while VDR expression was significantly increased in the STAT3-knockdown tumours.

\section{Discussion}

As a transcriptional repressor, $E Z H 2$ is overexpressed and has been regarded as a novel candidate oncogene in several types of human cancer [8,38,39]. It has been reported that overexpression of EZH2 correlates with increased invasion in several cancers $[12,13]$. However, the abnormalities of EZH2 and its underlying mechanisms in the pathogenesis of CRC are not fully understood. Four lines of argument suggest that EZH2 plays an important role in CRC cell motility and metastasis. First, the expression of EZH2 was significantly increased in CRC cancer tissues and cell lines; second, overexpression of EZH2 in CRC tissues may increase the aggressiveness of the tumour; third, downregulation of $E Z H 2$ significantly decreased the cell invasive ability and secretion of MMP2 and MMP9 in CRC cells; and finally, overexpression of EZH2 markedly increased the metastatic ability of CRC cells and decreased overall survival in an in vivo model of CRC metastasis. Conversely, down-regulation of EZH2 significantly decreased the metastatic ability of CRC cells and increased overall survival in vivo.

Overexpression of $\mathrm{EZH} 2$ has been shown to downregulate the expression of E-cadherin by increasing the H3K27 trimethylation level at the E-cadherin promoter in prostate cancer cells [35]. An inverse correlation has been demonstrated between $E Z H 2$ and runt-related transcription factor 3 (RUNX3) gene expression in gastric, breast, prostate, colon, and pancreatic cancer cell lines, and EZH2 may bind to the RUNX3 gene promoter and trimethylate the H3K27 mark [40]. $E Z H 2$ has also been reported to regulate cell migration/invasion via the regulation of E-cadherin and transforming growth factor beta 1 (TGF- $\beta 1$ ) expression in ovarian carcinoma [33]. However, the molecular mechanisms by which $E Z H 2$ regulates CRC cell migration/invasion remain unclear. Vitamin D and VDR appear to mediate the prevention of tumour development by inducing cellular differentiation and inhibiting proliferation [41-43]. The expression of VDR is repressed in high-grade and metastatic colorectal cancer [44]. We found that the mRNA and protein levels of VDR were significantly up-regulated by knockdown of EZH2 expression in CRC cells (Figure 3). In luciferase assays, the transcriptional activity of the $V D R$ promoter was dramatically increased by down-regulating EZH2 (Figures 4A-4C). These data indicate that $E Z H 2$ may mediate VDR expression by regulating the transcriptional activity of the $V D R$ promoter in CRC cells. Our result is consistent with the report that $E Z H 2$ was predicted to regulate $V D R$ gene expression in germinal centre B cells [29]. Previous immunohistochemical studies have shown a reduction in the level of VDR in CRC samples [14,20,45]. We found that high $E Z H 2$ expression in human clinical specimens correlated with low expression of VDR (Spearman's correlation coefficient $r=-0.65720$; $p<0.0001$; Figure 4D). Furthermore, EZH2 -mediated CRC cell invasion depended on the down-regulation of VDR (Figures 3E and 3F). Down-regulation of VDR due to overexpression of $E Z H 2$ may present one pathway whereby EZH2 affects CRC cell invasion.

Previously we reported that the activation of STAT3 may mediate human CRC tumourigenesis and invasion [27], and STAT3 may directly induce cell invasion and participate in the development of resistance to chemotherapy and apoptosis during the epithelial-mesenchymal transition (EMT) stage of CRC progression [46]. As EZH2 was identified to participate in the invasion of CRC cells in the present study, we further examined whether STAT3 mediates the expression of $E Z H 2$ in CRC cells. We found that inhibition of STAT3 expression dramatically decreased EZH2 expression and increased VDR expression in CRC cells (Figures 5A and 5B). In addition, overexpression of STAT3 significantly increased EZH2 expression and decreased VDR expression in CRC cells (Figures 5C and 5D). These data demonstrate 


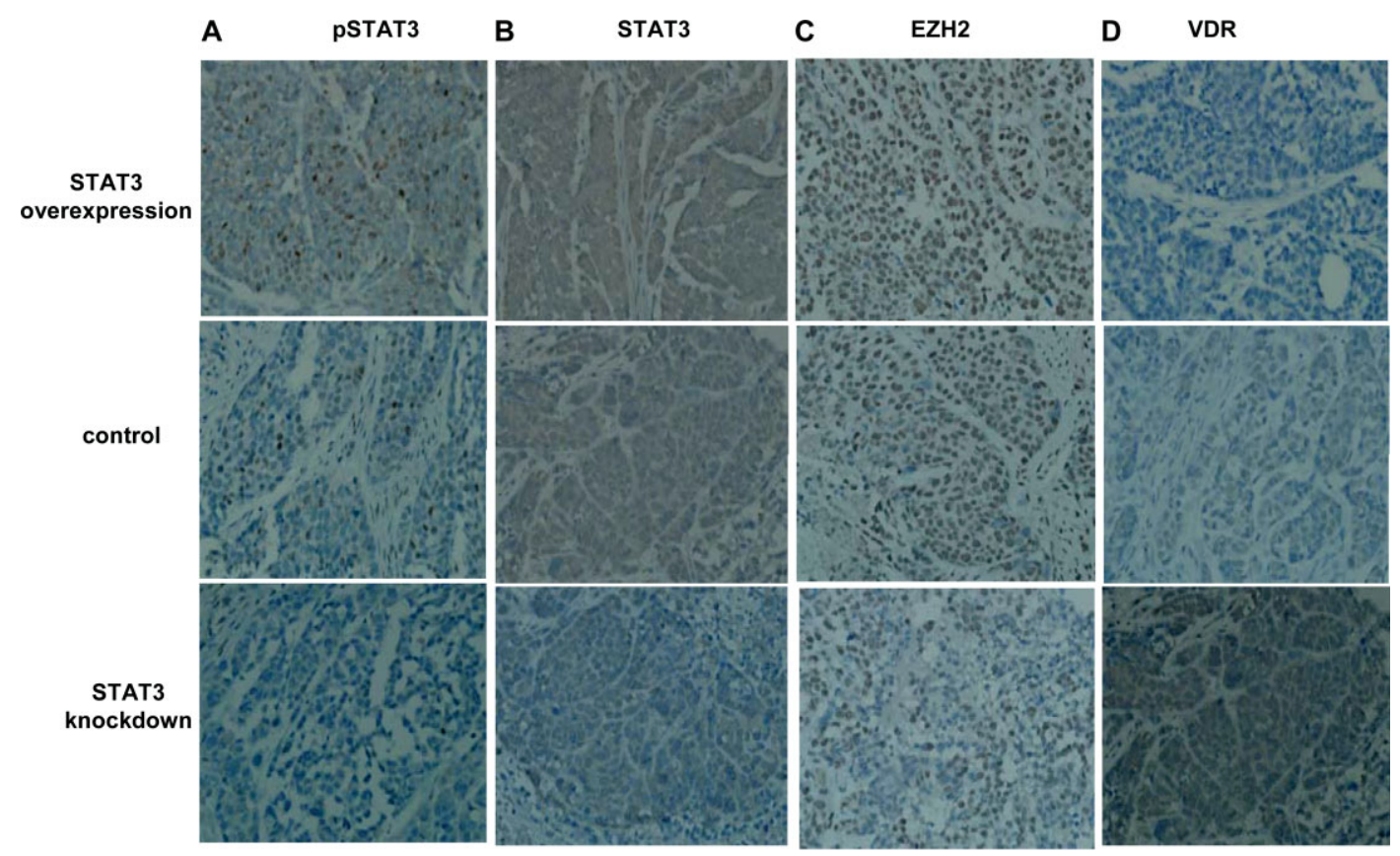

Figure 7. Expression levels of pSTAT3, STAT3, EZH2, and VDR in a nude mouse xenograft model of CRC. Immunohistochemical analysis of pSTAT3, STAT3, EZH2, and VDR in xenograft tissue sections. STAT3 was successfully up-regulated or down-regulated after introducing STAT3 shRNA and the STAT3 overexpression virus into nude mouse CRC cell model, respectively $(A, B)$. The level of pSTAT3 and the expression of EZH2 were significantly up-regulated in tumour samples with STAT3 overexpression (C), while the levels of pSTAT3 and EZH2 expression were significantly down-regulated in STAT3-knockdown tumour samples. The expression of VDR displayed an opposite trend (D). (A-D) Original magnification: $\times 400$.

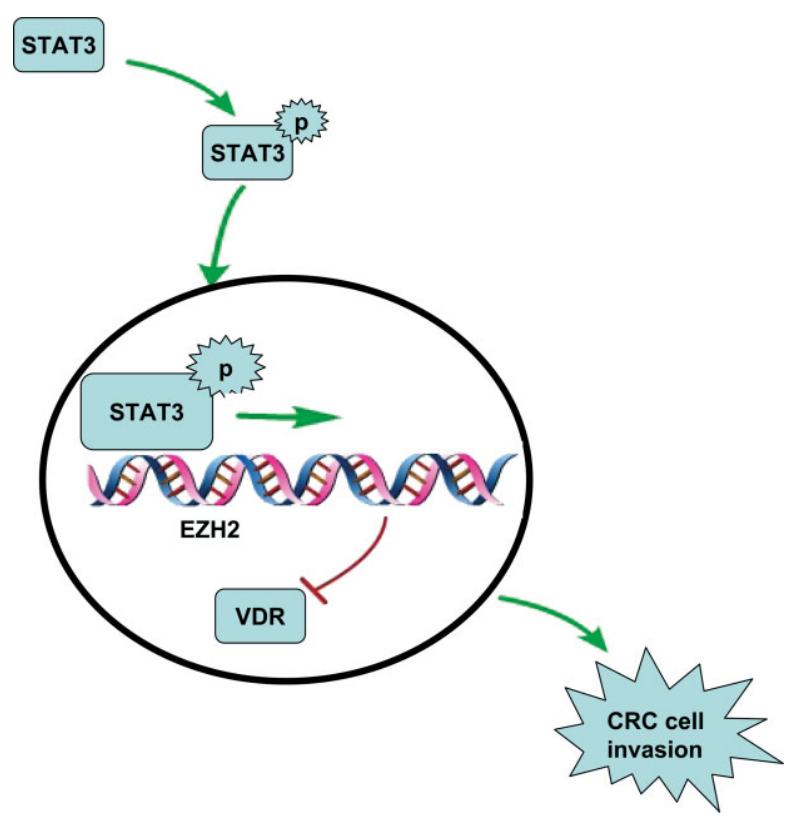

Figure 8. Model for the possible mechanism of EZH2-induced invasion in $\mathrm{CRC}$ and the regulatory mechanism of $E Z H 2$. Activation of STAT3 in CRC cells leads to overexpression of $E Z H 2$, which results in decreased expression of VDR and increased metastatic ability in CRC cells.

that STAT3 may regulate EZH2 and VDR expression in CRC cells. Moreover, EZH2 may play an important role in STAT3-induced VDR down-regulation and invasion in CRC cells (Figures 5E, 5F, and 6F). This finding is consistent with bioinformatics predictions that STAT3 may regulate EZH2 expression in prostate cancer cells [28]. Furthermore, a positive transcriptional effect of STAT3 binding the EZH2 promoter was shown in this study via luciferase and ChIP assays (Figures 6A-6E). However, VDR DNA was not detected in the ChIP assay using an antibody against STAT3 (data not shown), which suggests that STAT3 may directly bind to the EZH2 promoter, but not to the VDR promoter.

Our immunohistochemical data showed that pSTAT3 and $E Z H 2$ expression correlated positively with metastasis in human CRC, compared with control (Figure 6G and Table 1). In contrast, VDR expression was negatively associated with metastasis in human CRC (Figure 6G and Table 1). Moreover, a strong correlation between human CRC metastasis and EZH2 expression was illustrated in the multivariate analyses. Highly inverse correlations between the expression of EZH2 and pSTAT3 and that of VDR were also revealed in the animal model.

In conclusion, we found that EZH2 may play an important role in the process of tumour invasion in CRC. Overexpression of EZH2 may result in H3K27 trimethylation of the $V D R$ gene promoter, indicating that $V D R$ is a novel EZH2 target gene. EZH2 contributes to CRC cell invasion by suppressing VDR expression. Moreover, EZH2 overexpression can be induced by activated STAT3. EZH2 may mediate the STAT3-induced down-regulation of VDR and promote cell invasion in CRC (Figure 8). These findings may provide a potential target for treating aggressive colorectal cancers by inhibiting $E Z H 2$. 


\section{Acknowledgments}

This work was supported by grants from the National Natural Science Foundation of Key Program (No. 30830055), the Ministry of Public Health, China (No. 200802094), the Ministry of Education (No. 20090073110077) to FJY; the grant from the National Natural Science Foundation (No. 81001070) to SDF; the grant from the National Natural Science Foundation (No. 91129724), National Natural Science Foundation (No. 31271366) and Shanghai Rising-Star Program (No: 12QA1402000) to HJ.

\section{Author contribution statement}

Y-W Lin and L-L Ren performed all the experiments, analysed data, and wrote the manuscript. H Xiong, W Du, D-F Sun, Y-N Yu, T-T Sun, Y-R Weng, Y$\mathrm{C}$ Wang, Y Cui, J-L Wang, and $\mathrm{J} \mathrm{Xu}$ performed the experiments. Z-h Wang and H-y Chen analysed data. Z-G Han and N Shen provided technical support in the in vivo experiments. W Zou designed the experiments. $\mathrm{J}$ Hong and W Cao conceived and supervised the study, and wrote the manuscript. J-Y Fang designed and supervised this project and revised the manuscript.

\section{References}

1. Bates RC, Mercurio AM. The epithelial-mesenchymal transition (EMT) and colorectal cancer progression. Cancer Biol Ther 2005; 4: 365-370.

2. Markowitz SD, Dawson DM, Willis J, et al. Focus on colon cancer. Cancer Cell 2002; 1: 233-236.

3. Fearon ER. Molecular genetics of colorectal cancer. Annu Rev Pathol 2011; 6: 479-507.

4. van Engeland M, Derks S, Smits KM, et al. Colorectal cancer epigenetics: complex simplicity. J Clin Oncol 2011; 29: 1382-1391.

5. de Krijger I, Mekenkamp LJ, Punt CJ, et al. MicroRNAs in colorectal cancer metastasis. J Pathol 2011; 224: 438-447.

6. Kirmizis A, Bartley SM, Farnham PJ. Identification of the polycomb group protein $\mathrm{SU}(\mathrm{Z}) 12$ as a potential molecular target for human cancer therapy. Mol Cancer Ther 2003; 2: 113-121.

7. Sasaki M, Ikeda H, Itatsu K, et al. The overexpression of polycomb group proteins Bmi1 and EZH2 is associated with the progression and aggressive biological behavior of hepatocellular carcinoma. Lab Invest 2008; 88: 873-882.

8. Varambally S, Dhanasekaran SM, Zhou M, et al. The polycomb group protein EZH2 is involved in progression of prostate cancer. Nature 2002; 419: 624-629.

9. Simon JA, Lange CA. Roles of the EZH2 histone methyltransferase in cancer epigenetics. Mutat Res 2008; 647: 21-29.

10. Cao $\mathrm{R}$, Wang $\mathrm{L}$, Wang $\mathrm{H}$, et al. Role of histone $\mathrm{H} 3$ lysine 27 methylation in Polycomb-group silencing. Science 2002; 298: 1039-1043.

11. Kirmizis A, Bartley SM, Kuzmichev A, et al. Silencing of human polycomb target genes is associated with methylation of histone H3 Lys 27. Genes Dev 2004; 18: 1592-1605.

12. Fang J, Zhang M, Li Q. Enhancer of zeste homolog 2 expression is associated with tumor cell proliferation and invasion in cervical cancer. Am J Med Sci 2011; 342: 198-204.
13. Shin YJ, Kim JH. The role of EZH2 in the regulation of the activity of matrix metalloproteinases in prostate cancer cells. PLoS One 2012; 7: e30393.

14. Deeb KK, Trump DL, Johnson CS. Vitamin D signalling pathways in cancer: potential for anticancer therapeutics. Nature Rev Cancer 2007; 7: 684-700.

15. Haussler MR, Norman AW. Chromosomal receptor for a vitamin D metabolite. Proc Natl Acad Sci U S A 1969; 62: 155-162.

16. Palmer HG, Gonzalez-Sancho JM, Espada J, et al. Vitamin D(3) promotes the differentiation of colon carcinoma cells by the induction of E-cadherin and the inhibition of beta-catenin signaling. J Cell Biol 2001; 154: 369-387.

17. Vleminckx K, Vakaet L Jr, Mareel M, et al. Genetic manipulation of E-cadherin expression by epithelial tumor cells reveals an invasion suppressor role. Cell 1991; 66: 107-119.

18. Shabahang M, Buras RR, Davoodi F, et al. 1,25-Dihydroxyvitamin D3 receptor as a marker of human colon carcinoma cell line differentiation and growth inhibition. Cancer Res 1993; 53: 3712-3718.

19. Larriba MJ, Martin-Villar E, Garcia JM, et al. Snail2 cooperates with Snail1 in the repression of vitamin D receptor in colon cancer. Carcinogenesis 2009; 30: 1459-1468.

20. Evans SR, Nolla J, Hanfelt J, et al. Vitamin D receptor expression as a predictive marker of biological behavior in human colorectal cancer. Clin Cancer Res 1998; 4: 1591-1595.

21. Bachmann IM, Halvorsen OJ, Collett $\mathrm{K}$, et al. EZH2 expression is associated with high proliferation rate and aggressive tumor subgroups in cutaneous melanoma and cancers of the endometrium, prostate, and breast. J Clin Oncol 2006; 24: 268-273.

22. Fujii S, Tokita $\mathrm{K}$, Wada $\mathrm{N}$, et al. MEK-ERK pathway regulates $\mathrm{EZH} 2$ overexpression in association with aggressive breast cancer subtypes. Oncogene 2011; 30: 4118-4128.

23. Varambally S, Cao Q, Mani RS, et al. Genomic loss of microRNA101 leads to overexpression of histone methyltransferase EZH2 in cancer. Science 2008; 322: 1695-1699.

24. Gupta RA, Shah N, Wang KC, et al. Long non-coding RNA HOTAIR reprograms chromatin state to promote cancer metastasis. Nature 2010; 464: 1071-1076.

25. Spano JP, Milano G, Rixe C, et al. JAK/STAT signalling pathway in colorectal cancer: a new biological target with therapeutic implications. Eur J Cancer 2006; 42: 2668-2670.

26. Lassmann S, Schuster I, Walch A, et al. STAT3 mRNA and protein expression in colorectal cancer: effects on STAT3-inducible targets linked to cell survival and proliferation. J Clin Pathol 2007; 60: $173-179$.

27. Xiong $\mathrm{H}$, Zhang ZG, Tian XQ, et al. Inhibition of JAK1, 2/STAT3 signaling induces apoptosis, cell cycle arrest, and reduces tumor cell invasion in colorectal cancer cells. Neoplasia 2008; 10: 287-297.

28. Yeh HY, Cheng SW, Lin YC, et al. Identifying significant genetic regulatory networks in the prostate cancer from microarray data based on transcription factor analysis and conditional independency. BMC Med Genomics 2009; 2: 70.

29. Velichutina I, Shaknovich R, Geng H, et al. EZH2-mediated epigenetic silencing in germinal center $\mathrm{B}$ cells contributes to proliferation and lymphomagenesis. Blood 2010; 116: 5247-5255.

30. Langlois MJ, Bergeron S, Bernatchez G, et al. The PTEN phosphatase controls intestinal epithelial cell polarity and barrier function: role in colorectal cancer progression. PLoS One 2010; 5: e15742.

31. Samuels Y, Diaz LA Jr, Schmidt-Kittler O, et al. Mutant PIK3CA promotes cell growth and invasion of human cancer cells. Cancer Cell 2005; 7: 561-573.

32. Chang CJ, Yang JY, Xia W, et al. EZH2 promotes expansion of breast tumor initiating cells through activation of RAF1- $\beta$-catenin signaling. Cancer Cell 2011; 19: 86-100. 
33. Rao ZY, Cai MY, Yang GF, et al. EZH2 supports ovarian carcinoma cell invasion and/or metastasis via regulation of TGFbeta1 and is a predictor of outcome in ovarian carcinoma patients. Carcinogenesis 2010; 31: 1576-1583.

34. Tong ZT, Cai MY, Wang XG, et al. EZH2 supports nasopharyngeal carcinoma cell aggressiveness by forming a co-repressor complex with HDAC1/HDAC2 and Snail to inhibit E-cadherin. Oncogene 2012; 31: 583-594.

35. Cao Q, Yu J, Dhanasekaran SM, et al. Repression of E-cadherin by the polycomb group protein EZH2 in cancer. Oncogene 2008; 27: 7274-7284.

36. Huang C, Yang G, Jiang $\mathrm{T}$, et al. The effects and mechanisms of blockage of STAT3 signaling pathway on IL-6 inducing EMT in human pancreatic cancer cells in vitro. Neoplasma 2011; 58: 396-405.

37. Yadav A, Kumar B, Datta J, et al. IL-6 promotes head and neck tumor metastasis by inducing epithelial-mesenchymal transition via the JAK-STAT3-SNAIL signaling pathway. Mol Cancer Res 2011; 9: 1658-1667.

38. Raman JD, Mongan NP, Tickoo SK, et al. Increased expression of the polycomb group gene, $E Z H 2$, in transitional cell carcinoma of the bladder. Clin Cancer Res 2005; 11: 8570-8576.

39. Chen Y, Lin MC, Yao H, et al. Lentivirus-mediated RNA interference targeting enhancer of zeste homolog 2 inhibits hepatocellular carcinoma growth through down-regulation of stathmin. Hepatology 2007; 46: 200-208.
40. Fujii S, Ito K, Ito Y, et al. Enhancer of zeste homologue 2 (EZH2) down-regulates RUNX3 by increasing histone H3 methylation. $J$ Biol Chem 2008; 283: 17324-17332.

41. Shabahang M, Buras RR, Davoodi F, et al. Growth inhibition of HT-29 human colon cancer cells by analogues of 1,25dihydroxyvitamin D3. Cancer Res 1994; 54: 4057-4064.

42. Eisman JA, Barkla DH, Tutton PJ. Suppression of in vivo growth of human cancer solid tumor xenografts by 1,25-dihydroxyvitamin D3. Cancer Res 1987; 47: 21-25.

43. Spina CS, Ton $\mathrm{L}$, Yao $\mathrm{M}$, et al. Selective vitamin D receptor modulators and their effects on colorectal tumor growth. J Steroid Biochem Mol Biol 2007; 103: 757-762.

44. Matusiak D, Murillo G, Carroll RE, et al. Expression of vitamin D receptor and 25-hydroxyvitamin D3-1 $\alpha$-hydroxylase in normal and malignant human colon. Cancer Epidemiol Biomarkers Prev 2005; 14: $2370-2376$.

45. Ng K, Meyerhardt JA, Wu K, et al. Circulating 25-hydroxyvitamin $\mathrm{D}$ levels and survival in patients with colorectal cancer. J Clin Oncol 2008; 26: 2984-2991.

46. Xiong H, Hong J, Du W, et al. Roles of STAT3 and ZEB1 proteins in E-cadherin down-regulation and human colorectal cancer epithelial-mesenchymal transition. J Biol Chem 2012; 287: 5819-5832.

47. Gire V, Roux P, Wynford-Thomas D, et al. DNA damage checkpoint kinase Chk2 triggers replicative senescence. EMBO J 2004; 23: $2554-2563$.

\section{SUPPORTING INFORMATION ON THE INTERNET}

The following supporting information may be found in the online version of this article.

\section{Supplementary methods.}

Figure S1. (A) Western blot analysis data show that introduction of lenti-EZH2 shRNA-1/2 virus successfully decreased EZH2 expression in HCT116 CRC cells. (B) Western blot analysis data show that transduction of lenti-EZH2 overexpression virus remarkably increased the expression of EZH2 in SW1116 CRC cells.

Figure S2. The concentrations of MMP2 and MMP9 were tested by ELISA in control or EZH2 shRNA virus-infected CRC cells.

Figure S3. Deletion variants of the EZH2 promoter pGL3-1220, 1020, 820, 620, 420, 220, and 20 were derived from pGL3-1220.

Table S1. Clinicopathological characteristics of EZH2 expression in patients with CRC.

Table S2. Haematoxylin and eosin staining of lungs to evaluate the presence of lung metastasis in nude mice.

Table S3. Clinicopathological characteristics of pSTAT3 expression in patients with CRC.

Table S4. Clinicopathological characteristics of VDR expression in patients with CRC.

Table S5. Correlation among the expression of EZH2, pSTAT3 or VDR in colorectal cancers.

Table S6. Immunohistochemical staining of 27 cases of subcutaneously transplanted tumour in nude mice to evaluate the expression of pSTAT3, STAT3, EZH2, and VDR. 